\title{
Lemierre's Syndrome: Porphyromonas asaccharolytica as a Putative Pathogen
}

\author{
Shusuke Morizono, Munechika EnJoJI, Noriyuki SonODA, Marie FuKUshima, \\ Masami KuniYoshi, Kazuhiro KотоH, Makoto NAKAmUTA and Hajime NAWATA
}

\begin{abstract}
A case of Lemierre's syndrome is reported in which metastatic abscesses resulted from septic thrombophlebitis of the internal jugular vein secondary to bacterial pharyngitis. A 32-year-old male suffering from a painful left-sided neck mass, sore throat, and fever was admitted to our hospital. Computed tomography revealed thrombosis of the left internal jugular vein, septic pulmonary emboli, and a liver abscess. Blood culture showed Porphyromonas asaccharolytica. Although empyema occurred transiently during the treatment, the patient recovered following prolonged antimicrobial therapy. Although Fusobacterium species are a wellknown cause of Lemierre's syndrome, cases in whom Porphyromonas species was isolated have scarcely been reported. Moreover, case reports from Japan have been few.

(Internal Medicine 44: 350-353, 2005)
\end{abstract}

Key words: Lemierre's syndrome, thrombophlebitis, Fusobacterium, Porphyromonas

\section{Introduction}

Internal jugular vein thrombosis rarely causes painful neck masses. In 1936, Lemierre characterized and reported a distinctive syndrome of postanginal sepsis and internal jugular vein septic thrombosis (1). The following criteria for Lemierre's syndrome are generally accepted: 1) anaerobic primary infection in the oropharynx, 2) subsequent septicemia demonstrated by at least one positive blood culture, 3 ) evidence of thrombophlebitis of the internal jugular vein, 4) metastatic infections at one or more distant sites $(2,3)$. The syndrome has a propensity to strike previously healthy teen- agers and young adults. Initial symptoms of the syndrome are mainly nonspecific pain and swelling over the sternocleidomastoid muscle. The time of onset from the initial infection to the development of septicemia is typically 3 to 10 days. Septic embolization causes metastatic abscesses commonly in the lungs and less commonly in the large joints and the liver. In recent years, with widespread use of antibiotics to treat oral infections, this syndrome has become uncommon. Therefore, early diagnosis and treatment with effective antibiotic regimen can significantly reduce mortality and morbidity.

Over the past two decades, occasional case reports and literature reviews pertaining to Lemierre's syndrome have been published. The infection is caused mostly by Fusobacterium necrophorum and less commonly by other anaerobic organisms. However, there have been only a few case reports of the syndrome in Japan and only one case associated with Porphyromonas $(4,5)$. We describe a case with the classic clinical presentation and findings characteristic of Lemierre's syndrome. Isolates of $P$. asaccharolytica but not Fusobacterium were cultured from the patient's blood.

\section{Case Report}

A 32-year-old male with no significant past medical history presented to our hospital with a 4-day history of fever, sore throat, arthralgia, and neck pain restricting neck movements. Symptomatic treatment had been prescribed but there had been little response. Upon admission, he was febrile $\left(39.5^{\circ} \mathrm{C}\right)$ and tachycardic with tender left cervical lymphadenopathy. On physical examination, a painful neck mass on the left side was observed. The mass extended to the upper cervical and parieto-occipital regions. Typical rales were heard in the middle field of the right lung. Laboratory tests showed an increased high-sensitivity C-reactive protein (CRP) level of $21.32 \mathrm{mg} / \mathrm{dl}$ and neutrophilia $(91.7 \%$, white blood cell count; $6,830 / \mu \mathrm{l})$. Platelet count was $6.0 \times 10^{4} / \mu 1$.

From Department of Medicine and Bioregulatory Science, Graduate School of Medical Sciences, Kyushu University, Fukuoka

Received for publication July 7, 2004; Accepted for publication November 8, 2004

Reprint requests should be addressed to Dr. Munechika Enjoji, Department of Medicine and Bioregulatory Science, Graduate School of Medical Sciences, Kyushu University, 3-1-1 Maidashi, Higashi-ku, Fukuoka 812-8582 


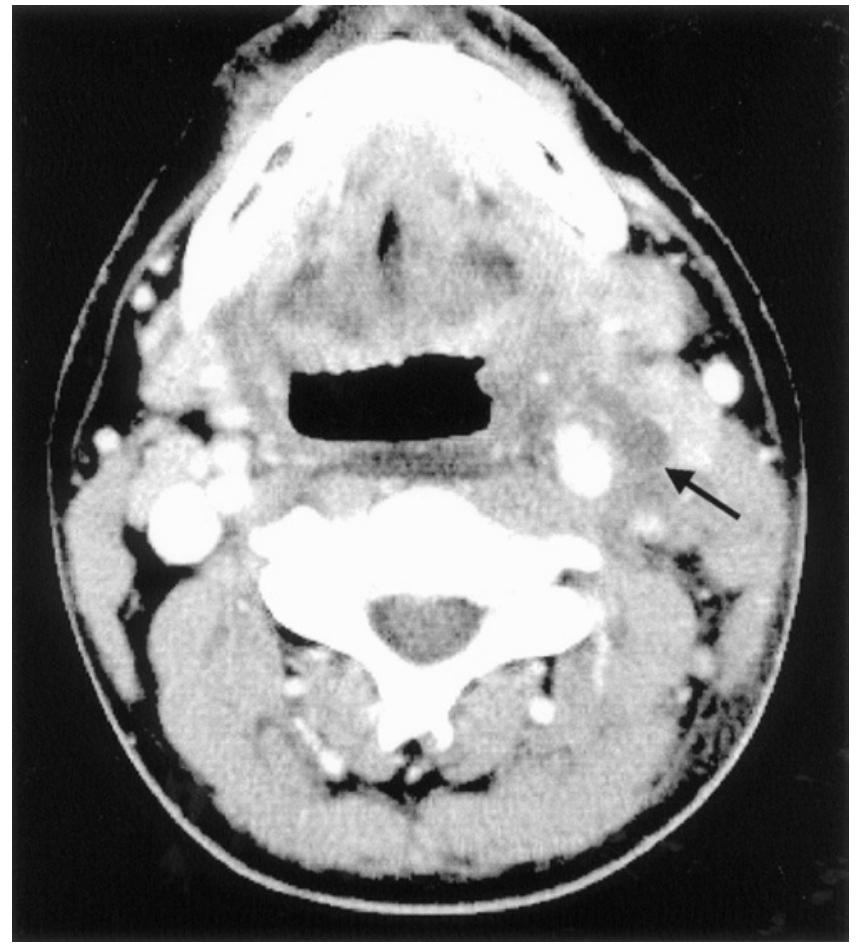

Figure 1. Contrast-enhanced CT image of cervical region demonstrating enlargement of left internal jugular vein. A hypodense lesion can be seen (arrows), which is thought to have occurred because of thrombosis of the vein. The left external jugular vein and right internal and external jugular veins appeared to be normal.

Coagulation test results were as follows: activated partial thromboplastin time, 38.1 seconds (control 27.5 seconds); prothrombin time, $62 \%$; fibrinogen, $760 \mathrm{mg} / \mathrm{dl}$; D-dimer, 7.5 $\mu \mathrm{g} / \mathrm{ml}$; FDP, $15.4 \mu \mathrm{g} / \mathrm{ml}$; and antithrombin III, 99\%. On the first day of admission, blood culture was examined in culture bottles, BacT/ALERT SA and SN (Biomérieux, Inc., Lyon, France), and the patient was treated with sulbactamcefoperazone $(4 \mathrm{~g}, 2 \mathrm{x})$ and meropenem $(2 \mathrm{~g}, 2 \mathrm{x})$. Because of the beginning of antimicrobial therapy, no more blood culture assays were performed. Chest radiograph demonstrated bilateral patchy infiltrates. Computed tomography (CT) of the neck revealed a filling defect of the left internal jugular vein consistent with thrombosis and there were abscesssuspected lesions in the parapharyngeal area (Fig. 1). Computed tomography (CT) of the chest demonstrated round opacities of both lungs and a small cavitary lesion was seen on the right-sided opacity in Fig. 2. On abdominal CT, an abscess-like low density area was found in the gallbladder bed of the liver (Fig. 3). According to these findings, a provisional clinical diagnosis of Lemierre's syndrome with septic disseminated intravascular coagulation (DIC) was made. After the diagnosis, intravenous administration of clindamycin $(2.4 \mathrm{~g}, 4 \times)$, meropenem $(2 \mathrm{~g}, 2 \times)$, and teicoplanin (400-800 mg, 1-2x) was started. Subsequently, inflam-



A

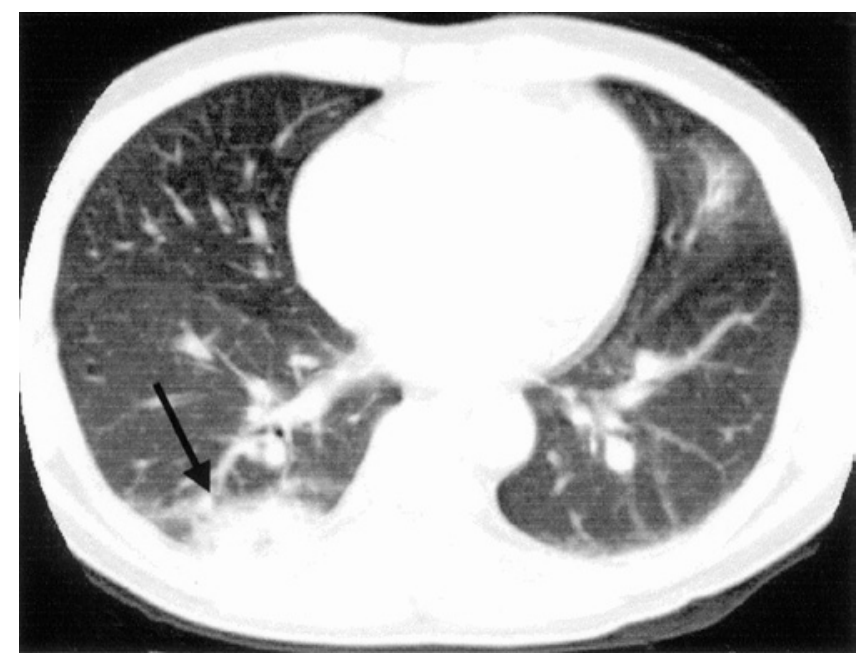

B

Figure 2. Chest CT image demonstrating round or patchy opacities (A and B, arrows) in the right lung consistent with septic emboli. Small cavitary lesions can be seen on the opacities.

matory markers gradually decreased and cultures of blood drawn at admission were positive for $P$. asaccharolytica by RapID Ana II testing (Remel, Inc., Lenexa, KS) which was sensitive to ongoing antibiotics. Isolated $P$. asaccharolytica was sensitive to ampicillin (ABPC), imipenem (IPM), cefazolin (CEZ), cefaclor (CCL), minocycline (MINO), chloramphenicol (CP), erythromycin (EM), clindamycin (CLDM), flomoxef (FMOX), cefmenoxime (CMX), cefminox (CMNX), cefdinir (CFDN), latamoxef (LMOX), levofloxacin (LVFX), fosfomycin (FOM), and azithromycin (AZM), and resistant to gentamycin (GM) and amikacin (AMK). Teicoplanin was administrated for the possibility of additional infection, e.g., methicillin resistant Staphylococcus aureus. Treatment for DIC consisted of gabexate mesilate and danaparoid.

During the second week of the treatment, however, the patient experienced bilateral pleuritic chest pain. Chest radiography at this stage revealed bilateral pulmonary infiltrates 


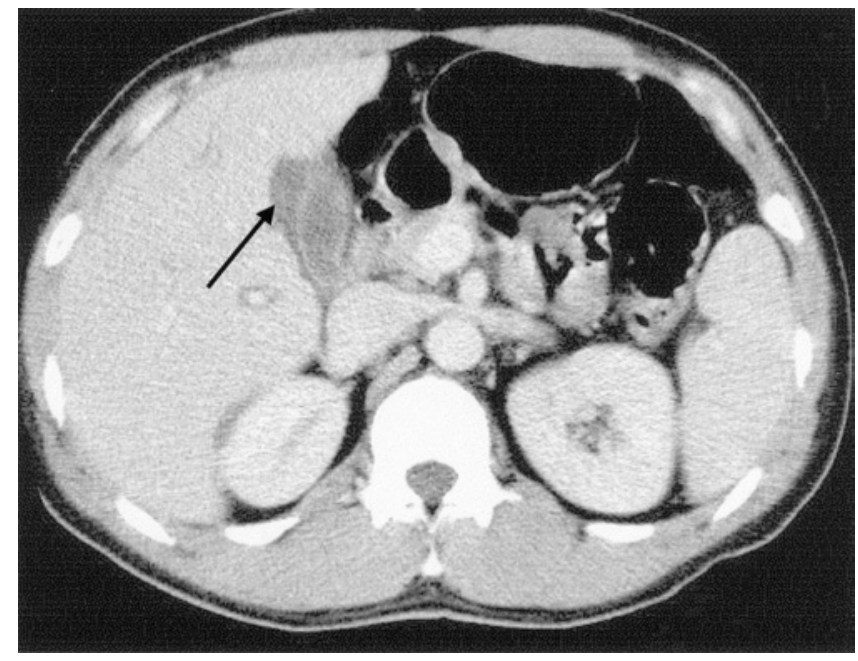

Figure 3. CT image of the upper abdomen revealed the presence of a low attenuation lesion in the liver (arrow).

with pleural effusion and CRP increased again (to 15.31 $\mathrm{mg} / \mathrm{dl})$. The effusion did not smell nasty and no bacteria were identified in effusion culture. The analysis of the effusion revealed empyema: cell count, $1 \times 10^{4} / \mu \mathrm{l}$; specific gravity, 1.030; total protein, $4.5 \mathrm{mg} / \mathrm{dl}$; and $\mathrm{LDH}, 1,220 \mathrm{U} / l$. Therefore, the antibiotics regimen was changed to clindamycin $(2.4 \mathrm{~g}, 4 \times)$ and biapenem $(1.2 \mathrm{~g}, 2 \times)$ to which the isolated $P$. asaccharolytica were also sensitive. These treatments proved effective, and after 3-week chemotherapy, CRP gradually decreased, ultimately returning to below detectable levels. On CT scans on day 34, there was no evidence of thrombosis, abscess, or inflammation of the neck, chest, or abdomen. Anticoagulation therapy was changed to oral administration of warfarin. The patient improved and was discharged on day 48 .

\section{Discussion}

This patient presented symptoms that fit the generally accepted criteria for Lemierre's syndrome: occurrence in healthy children and young adults, previous oropharyngeal infection, septicemia following oropharyngeal infection, swelling and lateral neck tenderness, and metastatic abscesses of the lung and liver. Fusobacterium was not isolated from the patient's blood or other tissues, but $P$. asaccharolytica was isolated from blood culture. F. necrophorum and other Fusobacterium species have remained the most common pathogens in Lemierre's syndrome and accounted for over $90 \%$ of the cases in a recent review $(2,6,7)$. Both Fusobacterium and Porphyromonas species belong to the family Bacteroidaceae, which are anaerobic gram-negative rods found as part of the indigenous flora of the oropharynx, gastrointestinal tract, and genitourinary tract (8). The anaerobic gram-negative bacteria show a similar susceptibility pattern to antimicrobial agents (8).
Parapharyngeal invasion by infiltrates and lymphatic or tonsillar venous are central to the development of Lemierre's syndrome. It is unclear why these pathogens become invasive subsequent to local infections. Organisms of the family of Bacteroidaceae are considered to be associated with thromboembolic phenomena. They release a lipopolysaccharide (LPS) component, which has strong biologic activity, and a lipid A moiety that has been implicated in the promotion of coagulation $(6,9)$. The coagulation mechanism is platelet-independent and does not require terminal components of complement. Therefore, once inflammation of the pharynx occurs, coagulation may be promoted, devitalized tissue with impaired blood supply may provide the anaerobic environment for bacterial proliferation, local defense may be compromised, and invasion by pharyngeal flora may be augmented. Furthermore, it is thought that proteolytic enzymes produced by the bacteria are associated with the spread of the infection.

The most common pathogen of Lemierre's syndrome, $F$. necrophorum, is a very virulent anaerobe that is peculiar in its ability to invade as a primary pathogen without the presence of serious underlying disease, and may cause severe infection (6). F. necrophorum infection may take part in the impairment of mucosal and cutaneous defense mechanisms because many of its extracellular products have been identified and presumably play a role in its spread into the tissues and bloodstream. It produces leukocidin, hemolysin, hemagglutinin, and lipase $(6,10-12)$. Leukocidin assists in the destruction of leukocytes, impairs leukocyte migration, and protects other facultative pathogens from phagocytosis. A possible role of hemolysin may be to aid in creating an anaerobic environment by lysing the erythrocytes and thereby reduce oxygen transport to the site of infection. Hemagglutinin causes platelet aggregation without lysis, and septic thrombus formation, thereby contributing to the establishment of a favorable anaerobic environment for growth of $F$. necrophorum. It has been suggested that phospholipase A and lysophospholipase are the components responsible for the hemolytic activity.

Only $P$. asaccharolytica was isolated from this patient; however, it is unclear whether this was truly the main pathogen present. First, Porphyromonas species are not as virulent as $F$. necrophorum. Second, in some cases no organism is isolated from blood cultures. Third, organisms most commonly isolated with Fusobacteria are anaerobic bacilli including Porphyromonas species $(6,10,13)$. Lastly, within Porphyromonas species, $P$. gingivalis and $P$. endodontalis are encountered in the oral cavity (8). They are important pathogens in oral, dental, and bite infections, and may readily multiply in the head, neck, and lower respiratory tract. On the other hand, it is commonly accepted that $P$. asaccharolytica is prevalent in the urogenital and intestinal tracts and is important in infections arising in these regions (8).

A synergistic, pathogenic complex has been demonstrated between Fusobacterium species and other anaerobic bacteria in the formation of abscesses in animal models. The complex 
facilitates the growth of $F$. necrophorum by lowering the oxygen tension and creating an anaerobic environment (10, 13, 14). Therefore, a similar synergistic relationship might occur between $F$. necrophorum and $P$. asaccharolytica, and this might play a role in the pathogenesis of Lemierre's syndrome in the present patient.

The course of Lemierre's syndrome is usually protracted because of continuing septic embolization. However, the role of anticoagulation therapy is controversial. Some reports do not recommend anticoagulation because early clot dissolution may promote the risk of extending the infection and the risk for emboli and hemorrhage (15-18). Others suggest that anticoagulation, by enhancing resolution of the source of septic embolization, may expedite quicker recovery from the thrombophlebitis (19-21). We added anticoagulation therapy with gabexate mesilate and danaparoid because the patient showed signs of DIC. We cannot appraise the clinical significance of the treatment and the routine use of anticoagulation therapy remains controversial.

Lemierre's syndrome will continue to occur mainly in children and young adults even in the modern antibiotic era. We hope that this report will heighten awareness and facilitate the diagnosis of Lemierre's syndrome.

\section{References}

1) Lemierre A. On certain scepticaemias due to anaerobic organisms. Lancet 1: 701-703, 1936.

2) Sinave CP, Hardy GJ, Fardy PW. The Lemierre syndrome: suppurative thrombophlebitis of the internal jugular vein secondary to oropharyngeal infection. Medicine 68: 85-94, 1989.

3) Stallworth JR, Carroll JM. Lemierre's syndrome: new insights into an old disease. Clin Pediatr 36: 715-718, 1997.

4) Nakamura $S$, Sadoshima $S$, Doi $Y$, et al. Internal jugular vein thrombosis, Lemierre's syndrome; oropharyngeal infection with antibiotic and anticoagulation therapy-a case report. Angiology 51: 173-177, 2000.

5) Sasaki $Y$, Iwata $H$, Kinoshita M, Sumiya M. Lemierre syndrome with extensive cervical venous thrombosis and multiple pulmonary embolisms. Nihon Naika Gakkai Zasshi 89: 2174-2176, 2000 (in Japanese).

6) Hagelskjaer Kristensen L, Prag J. Human necrobacillosis, with empha- sis on Lemierre's syndrome. Clin Infect Dis 31: 524-532, 2000.

7) Alvarez A, Schreiber JR. Lemierre's syndrome in adolescent children - anaerobic sepsis with internal jugular vein thrombophlebitis following pharyngitis. Pediatrics 96: 354-359, 1995.

8) Jousimies-Somer HR, Summanen PH, Wexler H, et al. Bacteroides, Porphyromonas, Prevotella, Fusobacterium, and other anaerobic gramnegative bacteria. in: Manual of Clinical Microbiology. 8th ed. Murray PR, Baron EJ, Pfaller MA, et al. Eds. ASM Press, Washington, 2003: $880-901$.

9) Golpe R, Marin B, Alonso M. Lemierre's syndrome (necrobacillosis). Postgrad Med J 75: 141-144, 1999.

10) Tan ZL, Nagaraja TG, Chengappa MM. Fusobacterium necrophorum infections: virulence factors, pathogenic mechanism, and control measures. Vet Res Commun 20: 113-140, 1996.

11) Amoako KK, Goto Y, Shinjo T. Comparison of extracellular enzymes of Fusobacterium necrophorum subsp. funduliforme. J Clin Microbiol 31: 2244-2247, 1993.

12) Tan ZL, Nagaraja TG, Chengappa MM, Smith JS. Biological and biochemical characterization of Fusobacterium necrophorum leukotoxin. Am J Vet Res 55: 515-521, 1994.

13) Brook I, Walker RI. The relationship between Fusobacterium species and other flora in mixed infection. J Med Microbiol 21: 93-100, 1986

14) Smith GR, Thornton EA. Pathogenicity of Fusobacterium necrophorum strains from man and animals. Epidemiol Infect 110: 499-506, 1993.

15) Leugers CM, Clover R. Lemierre syndrome: postanginal sepsis. J Am Board Fam Pract 8: 384-391, 1995.

16) Lustig LR, Cusick BC, Cheung SW, Lee KC. Lemierre's syndrome: two cases of postanginal sepsis. Otolaryngol Head Neck Surg 112: 767-772, 1995 .

17) DeSena S, Rosenfield DL, Santos S, Keller I. Jugular thrombophlebitis complicating bacterial pharyngitis (Lemierre's syndrome). Pediatr Radiol 26: 141-144, 1996.

18) Seidenfeld SM, Sutker WL, Luby JP. Fusobacterium necrophorum septicemia following oropharyngeal infection. JAMA 248: 1348-1350, 1982.

19) Goldhagen J, Alford BA, Prewitt LH, Thompson L, Hostetter MK. Suppurative thrombophlebitis of the internal jugular vein: report of three cases and review of the pediatric literature. Pediatr Infect Dis J 7: 410-414, 1988.

20) Ahkee S, Srinath L, Huang A, Raff MJ, Ramirez JA. Lemierre's syndrome: postanginal sepsis due to anaerobic oropharyngeal infection. Ann Otol Rhinol Laryngol 103: 208-210, 1994.

21) Anand VK, Morrison WV. Thrombophlebitis of the jugular vein. Ear Nose Throat J 66: 64-68, 1987. 